\title{
Mass distribution of the Galaxy with VERA
}

\author{
Nobuyuki Sakai ${ }^{1}$, Mareki Honma ${ }^{1,2}$, Hiroyuki Nakanishi ${ }^{3}$, Hirofumi \\ Sakanoue $^{3}$, Tomoharu Kurayama ${ }^{3}$ and VERA project members \\ ${ }^{1}$ The Graduate University for Advanced Studies (Sokendai), Mitaka, Tokyo 181-8588, Japan \\ ${ }^{2}$ Mizusawa VLBI Observatory, NAOJ, Mitaka, Tokyo 181-8588 \\ ${ }^{3}$ Faculty of Science, Kagoshima University, 1-21-35 Korimoto, Kagoshima, Kagoshima 890-0065 \\ email: nobuyuki.sakai@nao.ac.jp
}

\begin{abstract}
We aim to reveal the mass distribution of the Galaxy based on a precise rotation curve constructed using VERA observations. We have been observing Galactic $\mathrm{H}_{2} \mathrm{O}$ masers with VERA. We here report one of the results of VERA for IRAS $05168+3634$. The parallax is $0.532 \pm$ 0.053 mas which corresponds to a distance of $1.88_{-0.17}^{+0.21} \mathrm{kpc}$, and the proper motions are $\left(\mu_{\alpha} \cos \delta\right.$, $\left.\mu_{\delta}\right)=(0.23 \pm 1.07,-3.14 \pm 0.28)$ mas $\mathrm{yr}^{-1}$. The distance is significantly smaller than the previous distance estimate of $6 \mathrm{kpc}$ based on a kinematic distance. This drastic change places the source in the Perseus arm rather than in the Outer arm. Combination of the distance and the proper motions with the systemic velocity provides a rotation velocity of $227_{-11}^{+9} \mathrm{~km} \mathrm{~s}^{-1}$ at the source assuming $\Theta_{0}=240 \mathrm{~km} \mathrm{~s}^{-1}$. The result is marginally slower than the rotation velocity at LSR with $\sim 1-\sigma$ significance, but consistent with previous VLBI results for six sources in the Perseus arm. We also show the averaged disk peculiar motion over the seven sources in the Perseus arm as $\left(U_{\text {mean }}, V_{\text {mean }}\right)=(11 \pm 3,-17 \pm 3) \mathrm{km} \mathrm{s}^{-1}$. It suggests that the seven sources in the Perseus arm are systematically moving toward the Galactic center, and lag behind the Galactic rotation with more than $3-\sigma$ significance.
\end{abstract}

Keywords. astrometry - ISM:individual (IRAS 05168+3634) — techniques: interferometric - VERA

\section{Introduction}

The rotation curve can be used to estimate mass for spiral galaxies. It has been revealed that there exist plenty of flat rotation curves beyond optical disks in external spiral galaxies. This indicates the existence of large quantities of dark matter in the outer region of galaxies. Today, a well-developed interferometer technique at radio wavelengths can be used to conduct galactic astrometry over a kilo-parsec scale (e.g. VERA, VLBA). To construct a rotation curve of the Milky Way with high accuracy, we have been using VERA to observe Galactic objects with $\mathrm{H}_{2} \mathrm{O}$ maser emission in the Galactic-outer region. In this paper, we report the observational results for IRAS $05168+3634$.

\section{Results \& Discussion}

Eleven VLBI observations with VERA between October 2009 and May 2011 yielded the parallax and the proper motions for IRAS $05168+3634$. The parallax is $0.532 \pm 0.053$ mas, corresponding to a distance of $1.88 \pm{ }_{0.17}^{0.21} \mathrm{kpc}$. The proper motions are $\left(\mu_{\alpha} \cos \delta\right.$, $\left.\mu_{\delta}\right)=(0.23 \pm 1.07,-3.14 \pm 0.28)$ mas $_{\mathrm{yr}^{-1}}$. The distance is significantly smaller than the previous estimate of the kinematic distance, being $6 \mathrm{kpc}$ (Molinari et al. 1999). Our result places the source in the Perseus arm rather than in the Outer arm. Combination of the distance and the proper motions with the systemic velocity provides a rotation velocity of $227_{-11}^{+9} \mathrm{~km} \mathrm{~s}^{-1}$ at the source assuming $\Theta_{0}=240 \mathrm{~km} \mathrm{~s}^{-1}$. The result shows 


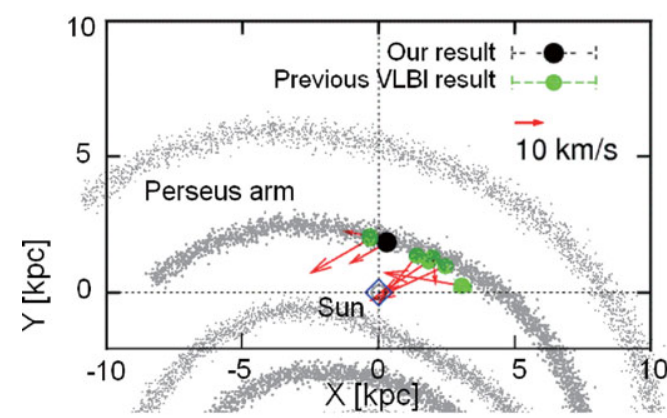

(a)

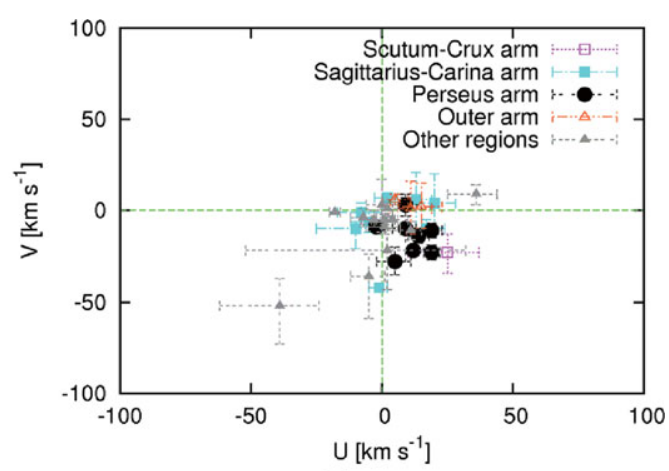

(b)

Figure 1. (a) Position of IRAS $05168+3634$ based on our observational result (black circle), while the green circles represent previous VLBI results in the Perseus arm. They are superposed on the Galactic-face on image (Georgelin \& Georgelin 1976). The solar position is (X, Y) = $(0,0) \mathrm{kpc}$. The red arrows show the peculiar motions. (b) Each source of Scutum-Crux (open square), Sagittarius-Carina (filled square), Perseus (circle), Outer arms (open triangle), and other regions (filled triangle) is plotted on the peculiar motions plane of $U$ and $V$ for 33 sources based on VLBI observations.

marginally slower rotation with respect to flat Galactic rotation $\left(\Theta(R)=\Theta_{0}\right)$. In addition, the slower rotation is almost consistent with previous VLBI results in the Perseus arm. Figure 1a shows the sources in the Perseus arm superposed on the Galactic face-on image (Georgelin \& Georgelin 1976). The black circle shows our result, while the green circles represent previous VLBI results. Figure $1 \mathrm{~b}$ also shows peculiar motions plane of $U$ and $V$ on which previous VLBI results are plotted. $U$ is directed toward the Galactic center, and $V$ is also directed toward the Galactic rotation. Obviously, sources in the Perseus arm (black circle) are systematically located in the lower right region of the $U-V$ plane. It means that the sources in the Perseus arm are systematically moving toward the Galactic center, and lag behind the Galactic rotation. We determine the averaged peculiar motions for seven sources in the Perseus arm as $\left(U_{\text {mean }}, V_{\text {mean }}\right)=(11 \pm 3,-17$ $\pm 3) \mathrm{km} \mathrm{s}^{-1}$. The result reveals the peculiar motions in the Perseus arm with more than 3- $\sigma$ significance. The peculiar motions may be caused at the inner edge of the Perseus arm where a shock front predicted by density-wave theory occurs (Mel'Nik et al. 1999).

\section{Acknowledgements}

NS acknowledges financial support from the SOKENDAI travel grant for overseas.

\section{References}

Georgelin, Y. M. \& Georgelin, Y. P. 1976, A\&A, 49, 57

Mel'Nik, A. M., Dambis, A. K., \& Rastorguev, A. S. 1999, Astron. Lett., 25, 518

Molinari, S., Brand, J., Cesaroni, R., \& Palla, F. 1996, A\&A, 308, 573 